(2) Open Access Full Text Article

\title{
Iron oxide-doped niosomes as drug carriers for magnetically targeted drug delivery
}

This article was published in the following Dove Press journal:

International Journal of Nanomedicine

\section{Ridhima Juneja \\ Indrajit Roy}

Department of Chemistry, University of Delhi, New Delhi, Delhi, India
Correspondence: Indrajit Roy Department of Chemistry, University of Delhi, New Delhi, Delhi I I0007, India Email indrajitroyl I@gmail.com

\begin{abstract}
Magnetism has wide applications in various fields, such as diagnostics, drug targeting, molecular biology, cell isolation, cell purification, hyperthermia, and radioimmunoassay. In this study, we synthesized niosomes doped with iron oxide nanoparticles and a fluorophore for potential applications in magnetically targeted drug delivery. Release kinetics of the fluorophore and cytotoxicity were assessed. The results demonstrate that niosomes doped with iron oxide nanoparticles can serve as proficient and effective drug carriers in magnetically targeted drug delivery.
\end{abstract}

Keywords: iron oxide nanoparticles, niosomes, fluorophores, drug carriers, magnetically targeted delivery

\section{Introduction}

Nanotechnology is the engineering of functional systems at the molecular scale. Nanoparticles are the simplest form of structures with sizes in the nanometer range. ${ }^{1}$ Vesicular systems are novel means of delivering drugs in a controlled manner to enhance bioavailability and obtain therapeutic effects over a longer period of time. ${ }^{2}$ Niosomes (NIO) are hydrated vesicular systems containing nonionic surfactants along with cholesterol or other lipids, delivering drug to targeted site. They are nontoxic, require less production cost, and are stable over a longer period of time in different conditions. Hence they overcome the drawbacks of liposomes.,

Magnetic nanomaterials, such as iron oxide (IO) nanoparticles, have applications in numerous biomedical fields, such as diagnostics, drug targeting, molecular biology, cell isolation, cell purification, hyperthermia, and radioimmunoassay. Combining the properties of the vesicular system with the magnetic targeting will provide us with a better tool for drug delivery.

\section{Materials and methods}

Polysorbate (Tween 80), cholesterol, Nile red, and MTT reagent (3-[4,5-dimethylthiazol2-yl]-2,5-diphenyltetrazolium bromide) were obtained from Sigma-Aldrich Co. (St Louis, MO, USA). Cell culture media (Dulbecco's Modified Eagle's Medium), fetal bovine serum, antibiotics, and amphotericin B were purchased from Thermo Fisher Scientific (Waltham, MA, USA). Human lung cancer A-549 cells were obtained from American Type Culture Collection (Manassas, VA, USA).

\section{Particle characterization}

\section{Transmission electron microscopy (TEM)}

For TEM, the aqueous dispersions of samples were sonicated, drop-coated, and dried to form a thin film on formvar-coated 200 mesh copper grids (Ted Pella, 


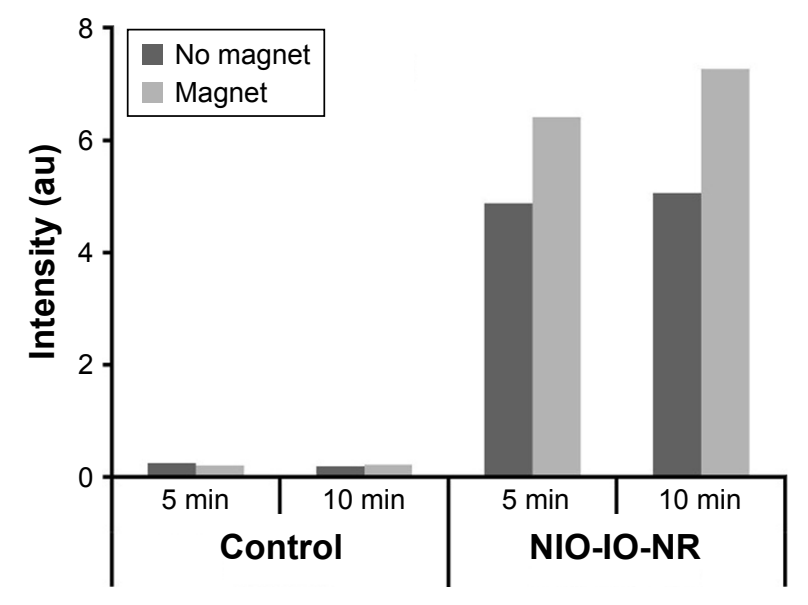

Figure I Time dependent cellular uptake shown for NIO-IO-NR, indicating an enhanced uptake in the presence of an external magnetic source.

Abbreviation: NIO-IO-NR, niosome encapsulating Nile red doped with iron oxide.

Redding, CA, USA). They were then negative stained using phosphotungstic acid, followed by imaging using a Tecnai G2-30 U Twin TEM instrument (FEI, Eindhoven, the Netherlands) with an acceleration voltage of $300 \mathrm{kV}$.

\section{Dynamic light scattering (DLS)}

The hydrodynamic diameters of the nanoparticles were further analyzed by DLS measurements. Aqueous dispersed nanoparticle samples were taken in glass cuvettes and analyzed using a NANO-ZS series Malvern Zetasizer instrument. A He-Ne laser (wavelength $633 \mathrm{~nm}$, power $4 \mathrm{~mW}$ ) was used as the light source.

\section{Optical studies}

\section{Ultra-violet-visible spectroscopy}

Absorption spectra of the synthesized nanoparticles were recorded using a Shimadzu UV-1601 spectrophotometer (Shimadzu, Kyoto, Japan).

\section{Fluorescence spectroscopy}

Emission spectra of the prepared niosomal nanoparticles (with and without dye and iron oxide [IO]) were recorded using a Cary Eclipse fluorescence spectrometer (Varian, Palo Alto, CA, USA).

\section{Stability studies}

The stability of the fluorophore NR, both free and nanoencapsulated, was studied using a fluorescence quenching experiment. The fixed concentration of the fluorophore (free and nanoencapsulated) was then treated with various concentrations of the chemical quencher copper sulfate. ${ }^{5}$

\section{Release kinetics}

The release patterns of the encapsulated fluorophore from the polymeric nanoparticles, with different size and surface properties, were examined by dialyzing them against a solution containing Tween 80 micelles ( $1 \%$ in water) for 2 weeks.

\section{In vitro studies}

For carrying out in vitro studies for the nanoparticles, lung carcinoma (A-549) cells were used. The cells were grown under standard cell culture conditions of $37^{\circ} \mathrm{C}, 5 \% \mathrm{CO}_{2}$ in a humidified incubator.

\section{Cytotoxicity}

For analyzing cell viability upon treatment with nanoparticles, MTT assay was carried out using MTT reagent (5 mg/mL in phosphate-buffered saline). ${ }^{6}$

\section{Cellular uptake}

For monitoring the uptake of the dye-doped nanoparticles in cells, fluorescence microscopy was carried out using a fluorescent Nikon TS-100 inverted microscope and photographed using a Nikon Digital Sight DS-Fil Camera (Nikon, Tokyo, Japan).

For a quantitative determination of the cellular uptake of the doped nanoparticles, analysis of the cell lysate was carried out. The effect of an external magnet on cellular uptake was also probed.

\section{Results and discussion}

The average sizes of NIO and NIO-doped IO (NIO-IO) found using TEM were in the range of $130-190 \mathrm{~nm}$. The mean hydrodynamic diameters of NIO and NIO-IO were found by DLS and were 168 and $176 \mathrm{~nm}$, respectively. The synthesized particles were slightly polydispersed.

The absorption and emission spectra for dye-doped NIO showed an efficient encapsulation. Being hydrophobic in nature, the fluorophore does not show fluorescence in aqueous solution. However, when it was encapsulated in aqueous dispersion of NIO, an appreciable amount of fluorescence was observed, proving the successful encapsulation in the lipophilic niosomal bilayer. In addition, iron oxide doped dye encapsulating NIO retained appreciable fluorescence, with a slight drop in the same, which was attributed to the quenching of dye due to the presence of metal ions.

We next investigated whether nanoencapsulation enhanced the stability of fluorophores, using a chemically induced fluorescence quenching experiment. The result 
indicated that the optical stability of the fluorophore was increased upon nanoencapsulation.

The release profile of encapsulated molecules from carrier nanoparticles showed complete release of the dye within a time span of 2 weeks, thereby making this system an efficient carrier for drug delivery.

Finally, we investigated the interaction of these nanoparticles with cells in vitro. The cellular uptake studies, both qualitative and quantitative, indicated that these nanocarriers are taken up by the cells in significant amounts. This uptake was enhanced under the influence of magnetic field. Lastly, both placebo (NIO) and dye-doped NIO (niosome encapsulating Nile red) (Figure 1), along with NIO-IO, were found to be non-cytotoxic. Thus, these carriers can work as efficient drug delivery vehicles without causing any toxicity to the normal cells.

\section{Conclusion}

The studies carried out indicate a proficient and effective application of the iron-oxide doped niosomes for magnetically targeted drug delivery.

\section{Acknowledgment}

The authors gratefully acknowledge the funding from the University Grant Commission (UGC), New Delhi.

\section{Disclosure}

The authors report no conflicts of interest in this work.

\section{References}

1. Prasad PN. Introduction to Biophotonics. New York, NY: Wiley; 2004

2. Kumar GP, Rajeshwarrao P. Nonionic surfactant vesicular systems for effective drug delivery an overview. Acta Pharma Sin B. 2011;1(4): 208-219.

3. Sankar V, Ruckmani K, Jailan S, Ganesan KS, Sharavanan S. Niosome drug delivery system: advances and medical applications an overview. Pharmacol Online. 2009;2:926-932.

4. Mahale NB, Thakkar PD, Mali RG, Walunj DR, Chaudhari SR. Niosomes: novel sustained release nonionic stable vesicular systems - an overview. Adv Colloid Interface Sci. 2012;183-184:46-54.

5. Juneja R, Roy I. Surface modified PMMA nanoparticles with tunable drug release and cellular uptake. RSC Adv. 2014;4:44472-44479.

6. Roy I, Ohulchanskyy TY, Pudavar HE, et al. Ceramic-based nanoparticles entrapping water-insoluble photosensitizing anticancer drugs: a novel drug-carrier system for photodynamic therapy. $\mathrm{JAm}$ Chem Soc. 2003;125(26):7860.
International Journal of Nanomedicine

\section{Publish your work in this journal}

The International Journal of Nanomedicine is an international, peerreviewed journal focusing on the application of nanotechnology in diagnostics, therapeutics, and drug delivery systems throughout the biomedical field. This journal is indexed on PubMed Central,

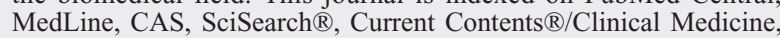

\section{Dovepress}

Journal Citation Reports/Science Edition, EMBase, Scopus and the Elsevier Bibliographic databases. The manuscript management system is completely online and includes a very quick and fair peer-review system, which is all easy to use. Visit http://www.dovepress.com/ testimonials.php to read real quotes from published authors. 